\title{
Evidências de validade da Escala de Orientação para a Comparação Social (INCOM) para o contexto de adolescentes portugueses
}

\author{
Samuel Lincoln Bezerra Lins ${ }^{1}$, Miguel Campos ${ }^{2}$, Ana C. Leite ${ }^{3}$, Catarina L. Carvalho ${ }^{2}$, \\ Sónia Cardoso ${ }^{2}$, Jean Carlos Natividade ${ }^{1}$ \\ ${ }^{1}$ Pontifícia Universidade Católica do Rio de Janeiro, Brasil \\ 2 Universidade do Porto, Portugal \\ 3 University of Roehampton, Reino Unido
}

\begin{abstract}
Resumo: Neste estudo procuramos validar a escala Iowa-Netherlands Comparison Orientation Scale numa amostra de adolescentes portugueses. Esta escala mede níveis de orientação da comparação social. Participaram no estudo 238 estudantes portugueses, com idades entre os 13 e os 18 anos $(M=15.43, D P$ = 1.76). Verificou-se que a estrutura da escala estava de acordo com a versão original do instrumento, dividindo-se em duas dimensões com índices satisfatórios de consistência interna: Opiniões e Aptidões. A dimensão Opiniões correlacionou-se positivamente com a Autoestima e o Materialismo, e a dimensão Aptidões apresentou correlações positivas com o Self-comparado, o Materialismo e a Impulsividade na compra. No que diz respeito às diferenças de sexo, as raparigas apresentaram médias superiores na dimensão de Opiniões e os rapazes em Aptidões. Os resultados confirmam a adequação da versão portuguesa da escala para avaliar a tendência para a comparação social em adolescentes.
\end{abstract}

Palavras-chave: Comparação Social; Adolescentes; Testes psicológicos; Validade do teste.

Validity evidence for Scale for Social Comparison Orientation (INCOM) in the Portuguese adolescents' context: The aim of this study was to translate and search for validity evidence of IowaNetherlands Comparison Orientation Scale for adolescents' Portuguese context. This scale assesses levels of social comparison orientation. Participants were 238 adolescents, with ages between 13 and 18 years $(M=15.43, S D=1.76)$. We verified that scale structure was in line with the original instrument version. Two dimensions emerged: Opinions and Abilities, both with satisfactory internal consistency indices. The dimension Opinions correlated positively with Self-esteem and Materialism; Abilities showed positive correlations with Self-compared, Materialism and Impulse buying. Regarding sex differences, girls showed higher mean for Opinions; boys higher mean for Abilities. Our results strengthen the Portuguese scale version adequacy to assess a tendency towards social comparison in adolescents.

Keywords: Social Comparison; Adolescents; Psychological test; Test validity.

Desde meados do século XX que é reconhecido o papel fundamental dos processos de comparação social, não só como um meio através do qual as pessoas compreendem melhor o mundo que os rodeia, mas também enquanto forma de avaliação do seu valor pessoal. Neste sentido, vários instrumentos de medida têm sido desenvolvidos em torno deste conceito.

Apesar de existirem diversas escalas adaptadas para o contexto português que recorrem ao conceito de comparação social, como a Escala de Comparação Social (Allan \& Gilbert, 1995), a Escala de Comparação Social através da Aparência Física (Ferreira, Gouveia, \& Duarte, 2011), e a Adolescent Social Comparison Scale - Revised, ASCS-R (Irons \& Gilbert, 2005), nenhuma afere a orientação/tendência para a comparação social. Neste sentido, o presente artigo visa colmatar esta lacuna e proporcionar evidências da validade da Iowa-Netherlands Comparison Orientation Measure - INCOM (Gibbons \& Buunk, 1999). Esta escala pretende aferir a tendência dos indivíduos para se compararem com outros. Embora a INCOM seja extensamente utilizada na investigação sobre comparação social (e.g., Bogaerts \& Mario, 2013; Feinstein et al., 2013; Norvilitis \& Yingmei, 2013; Petersen et al., 2012; Ruiter \& Sheri, 2015), não foi ainda aplicada ao contexto adolescente português.

\footnotetext{
${ }^{1}$ Dados de contacto para correspondência: PUC-Rio, Departamento de Psicologia, Rua Marquês de São Vicente, 225, Gávea, Rio de Janeiro, 22543-900. E-mail: samuellins@puc-rio.br.
} 


\section{Teoria da Comparação Social}

Na base de toda a investigação feita sobre os processos de comparação social estão os postulados de Festinger (1954). Através da teoria da comparação social, o autor propôs que as comparações sociais são fontes privilegiadas de informação sobre nós mesmos, influenciando os nossos comportamentos, experiências e julgamentos (e.g., Corcoran, Crusious \& Mussweiler, 2011). Assim, a comparação social ocorre quando as pessoas avaliam as suas próprias características, comparando as suas aptidões e opiniões com as de outras pessoas (Festinger, 1954). Por meio desse processo, os indivíduos não só aumentam a confiança no seu próprio valor, como também reduzem a incerteza acerca do mundo que os rodeia (Festinger, 1950). As comparações sociais são, sobretudo, estabelecidas com pessoas percebidas como semelhantes. Estes alvos de comparação preferenciais permitem aos indivíduos obter informações mais apropriadas e informativas sobre si mesmos (Festinger, 1950). Deste modo, a comparação social com outros semelhantes permite aos indivíduos atribuir sentido a si mesmos e ao mundo que os rodeia (Buunk \& Gibbons, 2006).

Enquanto existem aspetos da realidade que podem ser testados fisicamente (e.g., se atirar um ovo ao chão, será que ele parte?), outros só podem ser aferidos recorrendo a outras pessoas (e.g., será que é bom usar roupa desta marca?). Sendo assim, o processo de comparação social será, também, um meio de obtenção de validação social (Festinger, 1954).

\section{Elementos, Motivação e Consequências da Comparação Social}

Qualquer aspeto do indivíduo pode ser objeto de comparação com os outros (Gibbons \& Buunk, 1999). A investigação sugere uma variedade de domínios, tais como a imagem corporal (Krayer, Ingledew, \& Iphofen, 2008), os hábitos alimentares (Wheeler \& Miyake, 1992), ou as opiniões e aptidões pessoais em relação às quais os indivíduos procuram validação junto de outros semelhantes (Gibbons \& Buunk, 1999). Relativamente às motivações subjacentes à comparação social, para além da motivação para a autoavaliação, as pessoas podem também estar motivadas para o autoaperfeiçoamento, i.e., procurar continuamente aperfeiçoar-se e melhorar as suas capacidades, aprendendo com os outros (Taylor \& Lobel, 1989). Assim, poderão procurar comparar-se com outros que considerem superiores na dimensão de comparação - comparação ascendente (Morse \& Gergen, 1970; cf. Myers \& Crowther, 2009). Poderão ainda estar motivadas para o autoengrandecimento, i.e., motivação para obter ou manter uma imagem positiva de si mesmos (Suls \& Miller, 1977; Gibbons \& Buunk, 1999). Dessa forma, os objetos de comparação social serão indivíduos considerados inferiores na dimensão de comparação - comparação descendente (Taylor \& Lobel, 1989; ver também Bonifield \& Cole, 2008; Myers \& Crowther, 2009). 0 objeto de comparação poderá, assim, variar de acordo com a motivação pessoal (Wood, 1989).

No que diz respeito às consequências do processo de comparação social, e apesar de algumas exceções (e.g., Buunk, Collins, Taylor, Van Yperen, \& Dakof, 1990), a maioria da investigação nessa área sugere que as comparações ascendentes têm maior probabilidade de provocar consequências negativas (e.g., baixa autoestima, inveja, frustração) nas pessoas (Morse \& Gergen, 1970; Marsh \& Parker, 1984). Pelo contrário, as comparações descendentes geram consequências e sentimentos positivos, especialmente em indivíduos sob stress (Wood, Taylor, \& Lichtman, 1985).

Segundo Festinger (1954), os indivíduos podem inibir as suas comparações sociais com os outros, em especial quando essas comparações têm consequências negativas. Assim, seria de esperar que os indivíduos não se comparassem com outros percebidos como superiores, uma vez que comparações desse tipo são frequentemente depreciativas para si mesmos e acarretam consequências negativas para a sua autoavaliação. Contudo, a literatura indica que nem sempre é o caso (e.g., Myers \& Crowther, 2009). Por exemplo, as mulheres fazem frequentemente comparações sociais ascendentes, geralmente associadas à aparência física, apesar das consequências e sentimentos negativos daí decorrentes (Leahey, Crowther, \& Mikelson, 2007; Bamford \& Halliwell, 2009). Também Heinberg e Thompson (1995) verificaram que as mulheres que mais internalizam as normas culturais relativas à beleza são as que mais se comparam com modelos profissionais, que são percebidas como objetos relevantes de comparação social. Ainda no que respeita à imagem corporal, as mulheres não procuram apenas comparar-se com outras semelhantes, mas também com imagens dissimilares e idealizadas de si mesmo (Strahan, Wilson, Cresman, \& Buote, 2006; ver também Lev-Ari, Baumgarten-Katz \& Zohar, 2014).

\section{Diferenças Individuais no Processo de Comparação Social}

De acordo com Gilbert, Giesler e Morris (1995), o processo de comparação social é um impulso universal, espontâneo e relativamente automático que está ligado à nossa constituição biológica. Apesar disto, as diferenças individuais fazem-se notar: nem todas as pessoas possuem a mesma predisposição para se compararem com outras (e.g., Buunk \& Dijkstra, 2014). De facto, o grau, a frequência e as consequências da comparação social variam (Buunk \& Gibbons, 2006; Gibbons \& Buunk, 1999). Para captar estas 
diferenças interindividuais na predisposição para efetuar comparações sociais, Gibbons e Buunk (1999) propuseram o conceito de orientação para a comparação social. De acordo com esses autores, pessoas com alta orientação para a comparação social tendem a pensar mais sobre si mesmas na presença de outros, a ser empáticas e sensíveis às necessidades dos outros, e a possuir uma baixa autoestima (para uma revisão, Buunk \& Gibbons, 2006; ver também Buunk \& Dijkstra, 2014). São também essas pessoas as mais afetadas pelos resultados das comparações sociais (Buunk, Zurriaga, Peíró, Nauta, \& Gosalvez, 2005; Van der Zee, Oldersman, Buunk, \& Bos, 1998). Há, contudo, uma etapa da vida em que a tendência para se comparar com outros semelhantes poderá ser mais vincada: a adolescência.

\section{Comparação Social nos Adolescentes}

A adolescência é uma fase determinante no desenvolvimento de competências importantes para a vida adulta (Palmonari, Pombeni, \& Kirchler, 1992). Trata-se de uma fase amplamente marcada por sentimentos de incerteza e de ambiguidade (Palmonari et al., 1992), e por tensões e conflitos (Coleman \& Hendry, 1990). Nesta fase de desenvolvimento, o grupo de pares assume um papel central, acompanhando a transição do adolescente, das relações familiares para outros relacionamentos sociais. 0 adolescente procura autonomia relativamente à sua família, substituindo o seu papel desta pela companhia dos grupos de pares, com os quais partilha experiências e desenvolve novos valores (Palmonari et al., 1992).

A pertença grupal é tão relevante que pode até influenciar o comportamento dos adolescentes, mesmo antes de pertenceram a um grupo (Newman, Lohman, \& Newman, 2007). De facto, os adolescentes podem alterar os seus comportamentos para serem aceites num grupo. Por exemplo, investigações têm mostrado que o consumo de tabaco está associado ao grupo de pares e à motivação para a aprovação social (Conrad, Flay, \& Hill, 1992; Friedman, Lichtenstein, \& Biglan, 1985).

Os adolescentes prestam, também, atenção às consequências das atitudes e comportamentos dos seus pares. Fiske (2004) destacou que os adolescentes aprendem as atitudes que são aceitáveis ao observarem as recompensas e punições de que os outros são alvo (e.g., mediante a roupa que usam, a música que ouvem, as convicções que defendem, entre outros). Essa ideia relaciona-se com a necessidade de aprovação social e de pertença a grupos sociais (Baumeister \& Leary, 1995). 0 sentimento de pertença e a perceção de sucesso no relacionamento com outros têm um impacto poderoso e altamente recompensador na autoestima dos indivíduos (Leary, Tambor, Terdal, \& Downs, 1995). Por outro lado, o sentimento de rejeição pode representar custos elevados para os adolescentes, e está relacionado com problemas ao nível da autorregulação, ansiedade e depressão (Baumeister, DeWall, Ciarocco, \& Twenge, 2005).

Nos adolescentes, os processos de comparação social têm vindo a ser associados à insatisfação corporal, sobretudo em raparigas (Jones, 2001; cf. Krayer, Ingledew, \& Iphofen, 2007). Mueller, Pearson, Muller, Frank e Turner (2010) conduziram um estudo longitudinal no qual evidenciaram que as adolescentes que se comparavam com pares semelhantes no que diz respeito ao aspeto físico reportavam mais tentativas de perda de peso (ver também Paxton, Schutz, Wertheim, \& Muir, 1999). Jones, Vigfusdottir e Lee (2004) mostraram que a influência do grupo de pares - quer positiva, através de conversas sobre a imagem corporal, quer negativa, através de críticas a essa imagem - está significativamente associada à internalização de ideais de beleza e à imagem corporal em adolescentes de ambos os sexos. Field, Cheung, Wolf, Herzog, Gortmaker e Colditz (1999) conduziram um inquérito em jovens adolescentes do sexo feminino, das quais $69 \%$ indicou que as fotografias de revistas influenciam os seus ideais corporais e $47 \%$ referiu que essas imagens influenciam a sua vontade de perda de peso.

Os bens materiais são, também, um aspeto de comparação relevante para os adolescentes. Por exemplo, Chan e Prendergast $(2007,2008)$ encontraram evidências de comparação social com os pares, bem como com figuras públicas. Os adolescentes que fazem comparações deste tipo acreditam mais que os bens materiais estão relacionados com o sucesso e a felicidade e, como tal, ocupam um papel central na vida. No mesmo sentido, Twenge e Kasser (2013) mostram que o materialismo é um dos valores que tem vindo a aumentar na população adolescente nas últimas gerações.

A comparação social parece, assim, assumir um papel determinante no desenvolvimento de atitudes e na alteração de comportamentos por parte dos adolescentes. Torna-se, portanto, evidente a relevância do desenvolvimento de instrumentos que meçam a orientação para a comparação social neste público.

\section{Instrumentos que avaliam a comparação social}

Diversas escalas que avaliam a comparação social têm sido adaptadas para aplicação ao contexto português. Por exemplo, a Escala de Comparação Social - ECS (Social Comparison Scale) desenvolvida por Allan e Gilbert (1995), foi traduzida e adaptada ao contexto português por Gato (2003). A escala é 
composta por 11 itens bipolares (e.g., Inferior-Superior, Rejeitado-Aceite), onde é solicitado aos participantes que se posicionem relativamente a como se sentem no relacionamento com os outros (Allan \& Gilbert, 1995).

Ferreira, Gouveia e Duarte (2011) desenvolveram uma Escala de Comparação Social através da Aparência Física (ECSAF), com uma amostra de 1728 portuguesas, de modo a "medir a perceção de atratividade, de hierarquia social e de ajustamento ao grupo de acordo com a perceção que os sujeitos têm da sua aparência física, avaliando a forma como se comparam com os outros a este nível" (Ferreira, Gouveia, \& Duarte, 2011, p. 315).

Recentemente, a Escala de Comparação Social para Adolescentes - Revista (Adolescent Social Comparison Scale - Revised, ASCS-R) de Irons e Gilbert (2005), foi traduzida e adaptada por Xavier, Cunha, Gouvei e Medeiros (2014), também adaptada da Escala de Comparação Social (ECS), através da qual os adolescentes se comparam com outros a partir de adjetivos bipolares.

Por fim, destaca-se a escala que foi o alvo deste estudo, a Iowa-Netherlands Comparison Orientation Measure (INCOM) desenvolvida por Gibbons e Buunk, (1999). Esta escala pretende medir as diferenças individuais na orientação para a comparação social. Apesar de a INCOM ser comummente utilizada e difundida na literatura (e.g. Buunk, Ybema, Gibbons, \& Ipenburg, 2001; Buunk et al., 2005; Carey, Henson, Carey, \& Maisto, 2007; Norvilitis \& Mendes-Da-Silva, 2013; Piko \& Gibbons, 2008; Van Der Zee et al., 1998), não existem, que seja do nosso conhecimento, estudos que validem a sua aplicação em Portugal e, especificamente, junto da população adolescente.

A INCOM é constituída por 11 itens, e foi desenvolvida com base numa amostra de norteamericanos e holandeses, tendo mostrado evidências de validade para um total de 22 amostras (administrada a 10 amostras nos Estados Unidos e 12 amostras na Holanda). A amostra inicial, com a população americana, foi constituída por 403 adolescentes. Os resultados obtidos através de análise fatorial revelaram a presença de dois fatores designados respetivamente por Aptidões (6 itens) e Opiniões (5 itens).

Uma vez que se verificou que a estrutura de apenas um fator era também viável, foi realizada uma análise confirmatória que indicou que a estrutura bi-fatorial se ajustava melhor do que a estrutura unifatorial. Assim, sugere-se ser mais adequado assumir a INCOM como um construto com dois fatores distintos e relacionados entre si (Gibbons \& Buunk, 1999). Relativamente à consistência interna da escala, verificou-se um valor de coeficiente alfa de .83, na escala original, e valores similares nas restantes amostras, variando entre .78 e .85 nas 10 amostras norte-americanas, e entre .78 e .84 nas 12 amostras holandesas.

Posteriormente, a escala foi traduzida e testada numa amostra espanhola (Buunk, Belmonte, Peiró, Zurriaga, \& Gibbons, 2005) em dois estudos distintos. 0 primeiro estudo decorreu com uma amostra de 212 estudantes universitários, e o segundo com 782 trabalhadores adultos. A análise da consistência interna da escala identificou valores satisfatórios de consistência interna, de .80 e .81, respetivamente. Outros estudos revelaram uma boa consistência interna da escala e a mesma estrutura bi-fatorial (Norvilitis \& Mendes-Da-Silva, 2013). Neste sentido, o presente estudo pretende testar a validade da Iowa-Netherlands Comparison Orientation Measure (INCOM) no contexto de adolescentes portugueses.

\section{MÉTODO \\ Participantes}

Participaram no estudo 238 adolescentes (117 rapazes e 121 raparigas), com idades compreendidas entre os 13 e os 18 anos $(M=15.4, D P=1.76)$, que frequentavam o $8 .^{\circ}(n=101), 10 .^{\circ}(n=34), 11 .^{\circ}(n=$

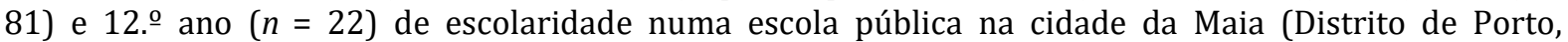
Portugal). A participação dos jovens foi voluntária e decorreu em contexto de sala de aula.

\section{Instrumentos}

Utilizou-se um questionário constituído por questões sociodemográficas (sexo, idade, escolaridade), a versão final em português da Iowa-Netherlands Comparison Orientation Measure (traduzida para este estudo), uma medida de autoestima (Avanci, Assis, Santos, \& Oliveira, 2007), uma medida de selfcomparado (Segabinazi, Giacomoni, Dias, Teixeira, \& Moraes, 2010), uma escala de materialismo (Richins, 2004), e uma escala de impulsividade nas compras (Sproles \& Kendall, 1986). De seguida, apresentamos uma descrição detalhada das escalas utilizadas. Todas as medidas foram recolhidas através de escalas de 7 pontos (1 = Discordo totalmente, 7 = Concordo totalmente $)$.

Iowa-Netherlands Comparison Orientation Measure

A INCOM (traduzida para português) foi originalmente desenvolvida por Gibbons e Buunk (1999) e pretende aferir os níveis de orientação para a comparação social por meio de duas dimensões: Aptidões e Opiniões. 0 instrumento consiste numa escala de 11 itens (ver Anexo). 
Medida de autoestima para adolescentes

A medida de autoestima inclui quatro itens, correspondentes à dimensão Alta Autoestima, do instrumento de autoestima de Avanci et al (2007). Os itens selecionados para este estudo foram os seguintes: "No global, eu estou satisfeito comigo; Sinto que tenho várias boas qualidades; Sou capaz de fazer coisas tão bem como a maioria das pessoas; Tenho uma atitude positiva em relação a mim mesmo". Neste estudo os itens apresentaram um índice de consistência interna igual a .86.

\section{Medida de self-comparado}

Esta escala (Segabinazi et al., 2010) corresponde a uma subescala do instrumento de satisfação de vida e sucesso em adolescentes por comparação com os seus pares. A subescala é composta por seis afirmações: "Meus amigos divertem-se mais do que eu; Meus amigos passeiam mais do que eu; Meus amigos podem fazer mais coisas do que eu; Os outros adolescentes ganham mais presentes do que eu; Os outros adolescentes têm mais amigos do que eu; Os outros adolescentes são mais alegres do que eu". Neste estudo, a subescala apresentou um índice de consistência interna de .86 .

\section{Escala de Materialismo}

Este instrumento (Richins, 2004) avalia a importância atribuída aos bens materiais e corresponde a uma versão de nove itens $(\alpha=.84)$ adaptada para português por Ponchio, Aranha e Todd (2007): "Admiro pessoas que possuem casas, carros e roupas caras; Gosto de gastar dinheiro com coisas caras; Minha vida seria melhor se tivesse muitas coisas que não tenho; Comprar coisas dá-me muito prazer; Ficaria muito mais feliz se pudesse comprar mais coisas; Gosto de muito luxo na minha vida; Incomoda-me quando não posso comprar tudo o que quero; Gastar dinheiro está entre as coisas mais importantes da vida; Gosto de possuir coisas que impressionam os outros."

\section{Escala de impulsividade na compra}

A escala, composta por três itens $(\alpha=.58)$, foi retirada do Consumer Style Inventory (CSI) (Sproles \& Kendall, 1986) e adaptada para o público adolescente no contexto brasileiro por Santos e Fernandes (2006): "Eu sou impulsivo(a) quando estou comprando; Frequentemente faço compras sem cuidado, as quais, mais tarde, desejaria não ter feito; Deveria planear as minhas compras com mais cuidado."

\section{Procedimentos}

Tradução da Escala

Para a tradução da escala para o português foram utilizadas a versão em inglês (Gibbons \& Buunk, 1999) e a versão em espanhol (Brunk et al., 2005). Optou-se por iniciar o processo de tradução usando a versão espanhola do instrumento em função da semelhança desse idioma com o português (comparativamente com o inglês). Inicialmente, duas psicólogas com conhecimentos avançados na língua inglesa e portuguesa, e fluentes em espanhol (língua nativa), traduziram, cada uma, o instrumento do espanhol para o português. De seguida, um dos investigadores compilou as duas versões num único documento, procurando consenso entre as traduções. Quando se encontraram diferenças entre as traduções, solicitou-se às tradutoras que, em conjunto, indicassem a redação mais adequada para o item.

Por fim, as tradutoras e um dos investigadores compararam a versão compilada em português com a versão em inglês a fim de adequarem a versão em português ao instrumento original. Após esse procedimento, realizaram-se alguns ajustamentos considerados pertinentes, obtendo-se a primeira versão do instrumento em português. Essa primeira versão do instrumento foi apresentada a um grupo de adolescentes para que avaliassem a percetibilidade e compreensão dos itens. A partir das sugestões do grupo, procedeu-se a pequenas alterações na redação de determinados itens (e.g., inclusão de pronomes na redação dos itens) e obteve-se a versão portuguesa da Iowa-Netherlands Comparison Orientation Measure (INCOM).

\section{Recolha e análise dos dados}

Os questionários foram aplicados em sala de aula, numa escola pública portuguesa. Os participantes foram informados sobre o caráter voluntário da participação, bem como da garantia do anonimato e sigilo da investigação. Os alunos que aceitaram participar no estudo responderam individualmente ao questionário. Inicialmente, excluíram-se doze questionários que continham valores omissos nos itens da INCOM. Em seguida, verificou-se a normalidade da distribuição dos dados olhando para os valores de assimetria e curtose, que revelaram ser inferiores a |1.41| e |1.98|, respetivamente. De acordo com Schumaker e Lomax (2004), estes valores são considerados aceitáveis. Além disso, segundo Gorsuch (1983), as análises fatoriais exploratórias e confirmatórias tendem a ser relativamente robustas contra ligeiras violações de normalidade. 


\section{RESULTADOS}

A fim de verificar a estrutura do instrumento em português, verificou-se inicialmente a fatorabilidade da matriz de correlação entre os itens, $K M O=.79$ e teste de esfericidade de Bartlett, $\chi^{2}(55, n=238)=$ 612.77, $p<.001$. Procedeu-se então à análise de componentes principais, com rotação Oblimin, uma vez que esse método oblíquo vai de encontro ao pressuposto teórico deste construto, de que as dimensões da comparação se correlacionam e, ao mesmo tempo, com o pressuposto de que esse método não impede que componentes ortogonais emerjam (cf. Pasquali, 2005).

A análise do gráfico scree plot de variâncias explicadas pelos valores próprios permitiu concluir que uma extração de dois componentes seria adequada (critério de Catell, 1966). Os dois componentes mostraram eigenvalues superiores a 1 (3.46 e 1.79), e explicam 47.72\% da variância dos dados. A correlação entre os componentes foi de .38. Por meio de uma análise paralela de eigenvalues aleatórios com 1000 amostras aleatórias considerando-se o mesmo $n$ e o número de itens deste estudo (critério de Horn, 1965), constatou-se que o último eigenvalue observado maior que o simulado foi o do segundo componente (terceiro componente: eigenvalue observado $=1.004$ e eigenvalue simulado $=1.23$ ). Além de a solução de retenção de dois componentes ser a mais adequada, de acordo com os critérios supracitados, essa solução também é a mais indicada tendo em conta a teoria sobre o construto e os resultados de estudos prévios com esse instrumento (e.g., Gibbons \& Buunk, 1999).

$\mathrm{Na}$ Tabela 1 encontram-se as saturações dos itens de cada dimensão extraída. Observa-se que os itens se agrupam em duas dimensões em concordância com a estrutura do instrumento original de Gibbons e Buunk (1999). As dimensões são denominadas Opiniões (itens 1, 7, 8, 9 e 10) e Aptidões (itens $2,3,4,5,6$ e 11). 0 primeiro componente, Opiniões, diz respeito à comparação dos sentimentos e pensamentos. 0 segundo componente, Aptidões, refere-se à comparação das suas capacidades. Ambos os componentes apresentam coeficientes alfa superiores a .70, considerados índices de consistência interna adequados para este instrumento (cf. Nunnally, 1978).

Adicionalmente, testou-se a estrutura por meio de uma análise fatorial confirmatória e comparouse o modelo de dois fatores com a estrutura uni-fatorial. Segundo Gibbons e Buunk (1999), a INCOM pode também ser aplicada como uma escala uni-fatorial, embora não seja recomendado. Para testar a melhor adequabilidade dos dados, utilizou-se a técnica de modelagem de equações estruturais (MES), utilizando o método da máxima verosimilhança. De facto, verificou-se que a solução com dois fatores apresenta índices de ajustamento mais adequados, $\chi^{2}(43, n=238)=123.36, p<.001 ; G F I=.91 ; C F I=.86 ; T L I=.82$; $R M S E A=.08$, do que a solução uni-fatorial, $\chi^{2}(44, n=238)=223.47, p<.001 ; G F I=.82 ; C F I=.68 ; T L I=$ $.61 ; R M S E A=.13$ (cf. Marôco, 2014: GFI, CFI e TLI > .80; RMSEA <.10). Neste sentido, para comparar os dois modelos, calculou-se o índice de ajustamento CAIC proposto por Byrne (2001), segundo o qual valores menores indicam um melhor ajustamento. Os resultados indicaram que o modelo de dois fatores $(C A I C=169.36)$ é mais adequado do que o modelo uni-fatorial $(C A I C=267.47)$.

Tabela 1. Solução Fatorial e Comunalidades dos Itens da Versão Portuguesa da INCOM (Rotação Oblimin)

\begin{tabular}{|c|c|c|c|}
\hline \multirow{2}{*}{ Item } & \multicolumn{2}{|c|}{ Componentes } & \multirow{2}{*}{ h2 } \\
\hline & Opiniões & Aptidões & \\
\hline 7. Gosto de conversar com os outros sobre as opiniões e experiências em comum. & .71 & .08 & .49 \\
\hline 8. Tento saber o que os outros pensam quando têm problemas semelhantes aos meus. & .80 & .10 & .63 \\
\hline 9. Gosto sempre de saber o que os outros fariam no meu lugar. & .76 & -.05 & .60 \\
\hline 10. Se quero saber mais sobre algo, tento saber o que os outros pensam sobre isso. & .61 & -.11 & .42 \\
\hline $\begin{array}{l}\text { 1. Eu comparo a maneira como as pessoas mais próximas de mim (por exemplo: família, } \\
\text { amigos, namorado(a)) se comportam em relação às outras pessoas. }\end{array}$ & .44 & -.35 & .38 \\
\hline $\begin{array}{l}\text { 2. Presto sempre muita atenção à maneira como faço as coisas, comparando-as com o modo } \\
\text { como os outros as fazem. }\end{array}$ & .26 & -.59 & .47 \\
\hline $\begin{array}{l}\text { 3. Se quero saber se o que estou a fazer está bem, comparo o que faço com o que os outros } \\
\text { fazem. }\end{array}$ & .17 & -.64 & .48 \\
\hline $\begin{array}{l}\text { 4. Eu comparo a maneira como me desenvolvo socialmente (por exemplo: habilidades sociais, } \\
\text { popularidade) em relação às outras pessoas. }\end{array}$ & .36 & -.51 & .46 \\
\hline 5. Não sou uma pessoa que se compara com as outras (invertido). & .27 & .75 & .55 \\
\hline 6. Comparo-me com os outros em relação ao que tenho conquistado na vida. & .26 & -.53 & .41 \\
\hline 11. Nunca comparo a minha condição de vida com a das outras pessoas (invertido). & .32 & .57 & .35 \\
\hline Número de itens & 5 & 6 & \\
\hline Valores próprios & 3.46 & 1.79 & \\
\hline \% Variância explicada & \multicolumn{2}{|c|}{47.72} & \\
\hline$M(\mathrm{DP})$ & $5.02(1.12)$ & $5.12(2.41)$ & \\
\hline Coeficiente Alfa & .74 & .74 & \\
\hline
\end{tabular}

Nota. Saturações maiores do que .40 estão a negrito. 
Em busca de evidências com base nas relações com outros construtos, testaram-se as relações entre as dimensões da orientação para a comparação social e outras variáveis apontadas como correlacionadas em estudos anteriores. Para esse efeito, calcularam-se os coeficientes de correlação de Pearson. A Tabela 2 apresenta os coeficientes obtidos. Destacam-se as correlações positivas entre ambos os componentes da orientação para comparação social e materialismo.

Tabela 2. Correlação dos Fatores da Escala de Comparação Social com Outras Escalas

\begin{tabular}{|c|c|c|c|c|c|}
\hline & 1 & 2 & 3 & 4 & 5 \\
\hline \multicolumn{6}{|l|}{ 1. INCOM - Opiniões } \\
\hline 2. INCOM - Aptidões & $.38^{* *}$ & & & & \\
\hline 3. Autoestima & $.16^{*}$ & -.07 & & & \\
\hline 4. Self-comparado & .03 & $.15^{*}$ & $-.32 * *$ & & \\
\hline 5. Materialismo & $.34^{* *}$ & $.43^{* *}$ & .05 & $.14^{*}$ & \\
\hline 6. Impulsividade nas compras & .10 & $.14^{*}$ & .08 & .08 & $.36^{* *}$ \\
\hline
\end{tabular}

Nota. $^{*} \mathrm{p}<.05,{ }^{* *} \mathrm{p}<.01$.

Por fim, no sentido de explorar o poder discriminativo do instrumento, realizou-se uma MANOVA sobre as componentes da INCOM comparando rapazes e raparigas. Os resultados mostraram diferenças de sexo na orientação para a comparação social, $\Lambda$ de Wilks $=.89, F(2,235)=15.07, p<.001, \eta^{2}=.11$. Enquanto as raparigas $(M=5.28, D P=1.02)$ tendem a comparar-se mais com as outras pessoas no que diz respeito aos sentimentos e pensamentos (Opiniões) do que os rapazes $(M=4.76, D P=1.17), F(1,236)=$ $13.50, p<.001, \eta^{2}=.05$, os rapazes $(M=5.43, D P=2.55)$ comparam-se mais relativamente às suas capacidades (Aptidões) do que as raparigas $(M=4.78, D P=2.23), F(1,236)=26.64, p<.001, \eta^{2}=.02$.

\section{DISCUSSÃo}

A aplicação da INCOM ao contexto de adolescentes portugueses apresentou bastantes semelhanças com a versão original da escala (Gibbons \& Buunk, 1999), bem como com a adaptação e validação para a população espanhola (Buunk et al., 2005). A estrutura de dois componentes do instrumento revelou-se consistente com a estrutura do instrumento original de Gibbons e Buunk (1999). Os autores da escala original reconhecem que uma solução uni-fatorial também obtém valores de ajuste aceitáveis. Contudo, no presente estudo, testou-se a adequabilidade dos dados a uma solução uni-fatorial e verificou-se que os valores de ajuste não são adequados. Este resultado reforça a ideia de que para medir a orientação para a comparação social é recomendado considerar ambos os fatores: Opiniões e Aptidões.

Os próprios autores da INCOM reforçam que estes fatores medem as "duas faces da mesma moeda" (Gibbons \& Buunk, 1999, p. 137). Nesse sentido, e tal como no estudo original, obteve-se uma associação significativa positiva entre ambas as dimensões. Deste modo, os resultados sugerem que será recomendado em estudos futuros considerarem-se ambos os componentes, no sentido de analisar a orientação para a comparação social em adolescentes portugueses.

Relativamente à relação entre a presente escala e restantes construtos, verificou-se uma correlação positiva, embora fraca, entre a dimensão de Opiniões e a autoestima dos participantes. Este resultado é, de certo modo, inesperado, uma vez que a correlação entre a autoestima e a INCOM na sua versão original é, consistentemente, negativa (Gibbons \& Buunk, 1999). Já na amostra espanhola, esta correlação é praticamente inexistente (Buunk et al., 2005). Este resultado poderá ser interpretado tendo em conta duas evidências: a primeira, consistente com Buunk et al (2005), sugere que os indivíduos possuem um autoconceito dinâmico, ajustável às diferentes situações sociais e que, portanto, em determinados contextos podem atribuir bastante valor às opiniões dos outros; a segunda, baseada nos pressupostos de Festinger $(1950,1954)$, prende-se com a premissa de que os indivíduos possuem uma necessidade inata de reduzir a incerteza. De facto, os indivíduos tendem a procurar as opiniões dos outros em situações de incerteza, procurando assim organizar e validar a sua realidade social. Neste sentido, não pode ser ignorado o período crítico que se vivia em Portugal na altura da recolha dos dados (Maio de 2011). 0 estudo decorreu num contexto de crise económica, com interferência direta de entidades externas, promovendo incerteza em relação ao futuro do país. Embora os adolescentes não sejam, tradicionalmente, alvos preferenciais de investigação acerca das consequências da crise, estudos recentes têm demostrado que são sensíveis a estas questões e que se preocupam com a crise (Lins \& Poeschl, 2015). Assim, é possível que, neste período particular de incerteza, a autoestima dos adolescentes estivesse mais suscetível à opinião dos outros.

Por outro lado, importa reforçar que a adolescência é, em si mesma, amplamente caracterizada por incerteza (Palmonari et al., 1992). Neste importante período do desenvolvimento, o grupo de pares 
assume uma importância central na vida do adolescente (e.g., Newman, Lohman, \& Newman, 2007), assumindo o importante papel de veículo de redução de incerteza. Como tal, é legítimo considerar que a incerteza decorrente desta fase de desenvolvimento, aliada à incerteza potencialmente criada por um período de instabilidade despoletada pela crise, poderá ter potenciado uma maior dependência da opinião dos outros e a consequente associação positiva entre estas opiniões e a autoestima. Contudo, estas interpretações deverão ser diretamente testadas em estudos futuros.

Constatou-se ainda uma relação positiva entre a dimensão de Aptidões e o self-comparado, mostrando que quanto mais os participantes estão orientados para se compararem com outros ao nível das aptidões, mais acreditam que os outros têm uma vida melhor. No entanto, esta correlação é relativamente baixa. Este resultado mostra que, para os adolescentes, as aptidões individuais têm pouco impacto no sucesso e na qualidade de vida dos outros, o que não é de todo surpreendente. Afinal, nesta fase da vida os adolescentes ainda são quase totalmente dependentes da família, pelo que uma grande parte da sua qualidade de vida depende dela.

Adicionalmente, obtiveram-se correlações positivas moderadas entre materialismo e ambas as dimensões da orientação para a comparação social ${ }^{2}$. Estes resultados devem ser lidos à luz das características da sociedade atual. Numa sociedade marcadamente consumista, os bens materiais são uma fonte de validação social e de estatuto, de tal modo que certos autores falam numa sobreposição entre o ter (i.e., os bens que se possui) e o ser (i.e., o valor atribuído ao indivíduo) (cf. Dittmar \& Pepper, 1994). Esta ideia torna-se, ainda, mais verdadeira junto do público adolescente da sociedade atual. Por exemplo, Twenge e Kasser (2013) mostraram que o materialismo nos adolescentes tem aumentado ao longo das gerações. Assim, e paralelamente, é possível que os adolescentes portugueses estejam orientados para fazer comparações sociais com base nos bens materiais que possuem.

Por fim, identificou-se a relação entre a impulsividade nas compras e orientação para a comparação social. Este é um resultado consistente com investigações anteriores, que indicam uma forte relação entre a comparação social e o comportamento de consumo (Karlsson, Gärling, Dellgran, \& Klingander, 2005). Esta relação é explicada mediante o desejo que os indivíduos possuem em obter produtos e artigos de outras pessoas, agindo, por isso, impulsivamente (Crusius \& Mussweiler, 2012).

A resposta dos participantes posicionou-se ligeiramente acima do ponto médio da escala. Contudo, esse valor é inferior à média obtida numa amostra de adolescentes americanos (Gibbons \& Buunk, 1999) ${ }^{3}$. Este resultado parece sugerir que existe uma maior orientação para a comparação social nos Estados Unidos da América do que em Portugal. De facto, quer a amostra holandesa (Gibbons \& Buunk, 1999) quer a amostra espanhola (Buunk et al., 2005) evidenciaram valores médios superiores à amostra americana na população adulta, sugerindo diferenças entre a cultura americana e a europeia. Assim, o presente estudo parece evidenciar que esta diferença transcultural tem início já na fase da adolescência. Apesar de não existirem ainda estudos transculturais que comparem a orientação para a comparação social em adolescentes, a investigação neste domínio tem demonstrado que, no contexto americano, a comparação social com os pares é forte (Jones et al., 2004; Mueller et al., 2010).

Relativamente às diferenças de sexo na orientação para a comparação social, verificou-se que rapazes e raparigas diferem quanto à dimensão que mais valorizam. As raparigas valorizam mais a componente Opiniões, enquanto os rapazes atribuem maior valor à dimensão Aptidões. Este resultado reflete os estereótipos de género vigentes na sociedade ocidental, na medida em que as raparigas parecem estar mais orientadas para obter validação com base naquilo que as outras pessoas pensam delas, enquanto os rapazes parecem obter essa validação com base em ações e competências. De facto, homens e mulheres desenvolvem uma identidade social diferente, na medida em que os homens procuram valorizar-se enquanto indivíduos únicos e independentes, e as mulheres enquanto grupo de pessoas interdependentes (Lorenzi-Cioldi, 1988).

Estas diferenças podem ter um impacto no comportamento de compra (Dittmar, 2011). Por um lado, os homens tendem a comprar objetos que reforçam a sua identidade enquanto sujeito independente, e que salientam a sua autonomia e capacidade para fazer coisas por si mesmo. Por outro lado, as compras das mulheres reforçam a sua identidade enquanto sujeito interdependente, e refletem a sua preocupação com os relacionamentos interpessoais. Pode ser, portanto, por este motivo que rapazes e raparigas estejam mais predispostos para diferentes dimensões da orientação para a comparação social.

Finalmente, a adequação desta escala para o contexto dos adolescentes portugueses pode potenciar a investigação e a intervenção em áreas do conhecimento nas quais a comparação social assume um papel preponderante, como a Psicologia e o Marketing. Por exemplo, partindo do pressuposto

\footnotetext{
${ }^{2}$ Cohen (1988) classifica correlações como: .10 a .29 (fraca); .30 a .49 (moderada); .50 a 1 (forte).

${ }^{3}$ No estudo com adolescentes americanos foi utilizada a escala original de 5 pontos. Ponderando para uma escala de resposta com 7 pontos, a média das suas respostas seria de 5.10, e portanto superior à média encontrada no presente estudo.
} 
de que as comparações sociais frequentes estão associadas a vários comportamentos e emoções destrutivos (e.g., White, Langer, Yariv, \& Welch IV, 2006; Jackson \& Chen, 2007) a intervenção psicológica poderá beneficiar dos avanços da investigação dedicada às comparações sociais. A associação deste construto com a autoestima poderá também ser preponderante na intervenção psicológica no contexto da saúde, por exemplo, nos transtornos alimentares, que têm registado uma incidência alta nos adolescentes, sobretudo do sexo feminino (e.g., Castro \& Goldstein, 1995). Sendo também a adolescência normalmente marcada pelo vínculo à escola, o uso de uma escala de comparações sociais pode revelar-se útil no estudo do processo de aprendizagem nos adolescentes (e.g., Blanton, Buunk, Gibbons, \& Kuyper, 1999). Ao nível do consumo, tendo em conta que as comparações sociais motivam o processo de compra, e com um crescente número de marcas a apostar no público-alvo dos adolescentes (e.g., Kang \& Park-Poaps, 2011), os instrumentos de medida das comparações sociais feitas pelos adolescentes ganham relevância.

\section{CONSIDERAÇÕES FINAIS}

Neste estudo, apresentámos evidências da validação da INCOM - escala de orientação para a comparação social - para o contexto dos adolescentes portugueses. Os resultados apontam para uma compreensão do conceito de orientação para a comparação social baseado nas dimensões de Opiniões e Aptidões, tal como proposto na escala original. Apesar da robustez dos dados, a utilização desta escala junto da população adolescente portuguesa deve ser encarada com a devida cautela, uma vez que não foi utilizado neste estudo uma amostra representativa.

De facto, a amostra deste estudo provém de uma escola pública de um grande centro urbano, não contemplando, assim, jovens adolescentes provenientes de meios sociais mais favorecidos e regiões geográficas menos desenvolvidas. Além disso, e dado que a evolução tecnológica das últimas décadas produz fortes mudanças sociais e psicológicas que se manifestam, não só, mas também, nos adolescentes, é possível que o conceito bidimensional de orientação para a comparação social fique desatualizado em relação ao originalmente proposto.

Apesar de a adolescência ser um período marcado por uma maior tendência para a manifestação de processos de comparação social, conforme evidenciado anteriormente, este é um conceito transversal a todas as faixas etárias. Assim, sugerimos que estudos futuros se foquem na adaptação e validação desta escala para outros públicos-alvo. Futuras investigações devem ainda incidir no melhor esclarecimento da validade discriminante desta escala. Neste estudo relacionou-se a INCOM com a autoestima, selfcomparado e materialismo, entretanto, algumas correlações encontradas podem ser consideradas fracas. Por outro lado, outros construtos que podem estar associados com a comparação social não foram considerados neste estudo (e.g., perceção da autoimagem corporal, ansiedade, depressão). Por fim, o motivo pelo qual rapazes e raparigas divergem face à importância atribuída às dimensões da orientação para a comparação social merece também atenção em investigações futuras.

\section{Referências}

Allan, S., \& Gilbert, P. (1995). A social comparison scale: Psychometric properties and relationship to psychopathology. Personality and Individual Differences, 19(3), 293-299. doi:10.1016/01918869(95)00086-L

Avanci, J., Assis, S., Santos, N., \& Oliveira, R. (2007). Adaptação transcultural de escala de auto-estima para adolescentes. Psicologia: Reflexão e Crítica, 20(3), 397-405. doi: /10.1590/s010279722007000300007

Bamford, B. \& Halliwell, E. (2009). Investigating the role of attachment in social comparison theories of eating disorders within a non-clinical female population. European Eating Disorders Review, 17(5), 371-379.

Bandura, A. (1965). Influence of models' reinforcement contingencies on the acquisition of imitative responses. Journal of Abnormal and Social Psychology, 1(6), 589-595. doi: 10.1037/h0022070

Bandura, A. (1977). Social learning theory. Englewood Cliffs: Prentice-Hall.

Baumeister, R., \& Leary, M. (1995). The need to belong: Desire for interpersonal attachments as a fundamental human motivation. Psychological Bulletin, 117I (3), 497-529. doi: 10.1037/00332909.117.3.497

Baumeister, R., DeWall, C., Ciarocco, N., \& Twenge, J. (2005). Social exclusion impairs self-regulation. Journal of Personality and Social Psychology, 88 (4), 589-604. doi: 10.1037/0022-3514.88.4.589

Blanton, H., Buunk, B.P., Gibbons, F., \& Kuyper, H. (1999). When better-than-others compared upward: choice of comparison and comparative evaluation as independent predictors of academic performance. Journal of Personality and Social Psychology, 76 (3), 420-430. doi: 10.1037/00223514.76.3.420 
Bogaerts, T., \& Mario, P. (2013). Less is more: Why some domains are more positional than others. Journal of Economic Psychology, 39 (C), 225-236. doi: 10.1016/j.joep.2013.08.005

Bonifield, C., \& Cole, C. A. (2008). Better him than me: social comparison theory and service recovery. Journal of the Academy of Marketing Science, 36(4), 565-577.

Buunk, A. P., \& Dijkstra, P. (2014). Social comparison orientation and perspective taking as related to responses to a victim. Psychology, 5, 441-450. doi: 10.4236/psych.2014.55054

Buunk, A., \& Gibbons, F. (2006). Social comparison orientation: A new perspective on those who do and those who don't compare with others. In S. Guimond (Ed.), Social comparison and social psychology; understanding cognition, intergroup relations and culture (pp. 15-33). Cambridge: Cambridge University Press.

Buunk, B., Belmonte, J., Peiró, J., Zurriaga, R., \& Gibbons, F. (2005). Diferencias individuales en la comparación social: Propiedades de la escala española de orientación hacia la comparación social. Revista Latinoamericana de Psicología, 37, 561-581.

Buunk, B., Collins, R., Taylor, S., VanYperen, N., \& Dakof, G. (1990). The affective consequences of social comparison: either direction has its ups and downs. Journal of Personality and Social Psychology, 59(6), 1238-49. doi: 10.1037/0022-3514.59.6.1238

Buunk, B., Zurriaga, R., Peíró, J., Nauta, A., \& Gosalvez, I. (2005). Social comparisons at work as related to a cooperative social climate and to individual differences in social comparison orientation. Applied Psychology: International Review, 54 (1), 61-80. doi: 10.1111/j.1464-0597.2005.00196.x

Buunk, B., Ybema, J., Gibbons, F., \& Ipenburg, M. (2001). The affective consequences of social comparison as related to professional burnout and social comparison orientation. European Journal of Social Psychology, 31 (4), 337-351. doi: 10.1002/ejsp.41

Byrne, B. (2001). Structural equation modeling with Amos: Basic concepts, applications and programming. New Jersey: Lawrence Erlbaum Associates.

Carey, K., Henson, J., Carey, M., \& Maisto, S. (2007). Which heavy drinking college students benefit from a brief motivational intervention? Journal of Consulting and Clinical Psychology, 75 (4), 663-669. doi:10.1037/0022-006X.75.4.663

Castro, J. M. \& Goldstein, S. (1995). Eating attitudes and behaviors of pre- and postpubertal females: clues to the etiology of eating disorders. Physiology \& Behavior, 58(1), 15-23. doi:10.1016/00319384(95)00027-G

Cattell, R. B. (1966). The scree test for the number of factors. Multivariate Behavioral Research, 1 (2), 245 276. doi: $10.1207 / \mathrm{s} 15327906 \mathrm{mbr} 0102 \_10$

Chan, K., \& Prendergast, G. (2007). Materialism and social comparison among adolescents. Social Behavior and Personality, 35(2), 213-228. doi: 10.2224/sbp.2007.35.2.213

Chan, K., \& Prendergast, G. (2008). Social comparison, imitation of celebrity models and materialism among Chinese youth. International Journal of Advertising, 27(5), 799-826. doi: 10.2501/S026504870808030X

Cohen, J (1988) Statistical power analysis for the behavioral sciences (2nd ed.). Hillsdale, NJ: Erlbaum.

Coleman, J., \& Hendry, L. (1999). The Nature of Adolescence. London: Routledge.

Conrad, K., Flay, B., \& Hill, D. (1992). Why children start smoking cigarettes: predictors of onset. British Journal of Addiction, 87 (12), 1711-1724. doi: 10.1111/j.1360-0443.1992.tb02684.x

Corcoran, K., Crusius, J., \& Mussweiler, T. (2011). Social comparison: Motives, standards, and mechanisms. In D. Chadee (Ed.), Theories in social psychology (pp. 119-139). Oxford: Wiley-Blackwell.

Crusius, J., \& Mussweiler, T. (2012). When people want what others have: the impulsive side of envious desire. Emotion, 12(1),142-53. doi: 10.1037/a0023523.

Cunha, M., Galhardo, A., \& Pinto-Gouveia, J. (2013). Child and adolescent mindfulness measure (CAMM): estudo das características psicométricas da versão portuguesa. Psicologia: Reflexão e Crítica, 26(3), 459-468. doi: 10.1590/S0102-79722013000300005

Feinstein, B., Hershenberg, R., Bhatia, V., Latack, J., Meuwly, N., \& Davila, J. (2013). Negative social comparison on Facebook and depressive symptoms: Rumination as a mechanism. Psychology of Popular Media Culture, 2 (3), 161-170. doi: 10.1037/a0033111

Ferreira, C., Pinto-Gouveia, J., \& Duarte, C. (2011). Desenvolvimento de uma escala de comparação social através da aparência física: Estrutura factorial e estudos de validação numa amostra feminina da população geral. Psychologica, 54, 309-330.

Festinger, L. (1950). Informal social communication. Psychological Review, 6, 271-282.

Festinger, L. (1954). A theory of social comparison processes. Human Relations, 7, 117-140. doi: $10.1177 / 001872675400700202$

Field, A., Cheung, L., Wolf, A., Herzog, D., Gortmaker, S., \& Colditz, G. (1999). Exposure to the mass media and weight concerns among girls. Pediatrics, 103, 36. doi: : 10.1542/peds.103.3.e36 
Fiske, S. (2004). Social beings: A core motives approach to social psychology. New York: Wiley.

Friedman, L., Lichtenstein, E., \& Biglan, A. (1985). Smoking onset among teens: An empirical analysis of initial situations. Addictive Behaviors, 10, 1-13. doi: 10.1016/0306-4603(85)90048-6

Gato, J. J. (2003). Evolução e ansiedade social (Dissertação de mestrado em Psicologia) Universidade de Coimbra, Coimbra.

Gibbons, F., \& Buunk, B. (1999). Individual differences in social comparison: Development and validation of a measure of social comparison orientation. Journal of Personality and Social Psychology, 76, 129142. doi: 10.1037/0022-3514.76.1.129

Gilbert, D., Giesler, R., \& Morris, K. (1995). When comparisons arise. Journal of Personality and Social Psychology, 69, 227-236. doi: 10.1037/0022-3514.69.2.227

Gorsuch, R. L. (1983). Factor analysis. Hillsdale, NJ: Erlbaum.

Heinberg, L., \& Thompson, J. (1995). Body image and televised images of thinness and attractiveness: A controlled laboratory investigation. Journal of Social \& Clinical Psychology, 14, 325-338. doi: 10.1521/jscp.1995.14.4.325

Horn, J. L. (1965). A rationale and test for estimating the number of factors in factor analysis. Psychometrika, 30, 179-185. doi: 10.1007/BF02289447

Irons, C., \& Gilbert, P. (2005). Evolved mechanisms in adolescent anxiety and depression symptoms: The role of the attachment and social rank systems. Journal of Adolescence, 28, 325-341. doi: 10.1016/j.adolescence.2004.07.004

Jackson, T., \& Chen, H. (2006). Identifying the eating disorder symptomatic in China: The role of sociocultural factors and culturally defined appearance concerns. Journal of Psychosomatic Research, 62, 241-249. doi: 10.1016/j.jpsychores.2006.09.010

Jones, D. (2001). Social comparison and body image: Attractiveness comparisons to models and peers among adolescent girls and boys. Sex Roles, 45, 645-664. doi: 10.1023/a:1014815725852

Jones, D., Vigfusdottir, T., \& Lee, Y. (2004). Body image and the appearance culture among adolescent girls and boys: An examination of friend conversations, peer criticism, appearance magazines, and the internalization of appearance ideals. Journal of Adolescent Research, 19, 323-339. doi: $10.1177 / 0743558403258847$

Kang, J., \& Park-Poaps, H. (2011). Motivational antecedents of social shopping for fashion and its contribution to shopping satisfaction. Clothing \& Textiles Research Journal, 29(4), 331-347. doi: 10.1177/0887302X11422443

Karlsson, N., Gärling, T., Dellgran, P., \& Klingander, B. (2005). Social comparison and consumer behavior: When feeling richer or poorer than others is more important than being it. Journal of Applied Social Psychology, 36, 1206-1222. doi: 10.1111/j.1559-1816.2005.tb02167.x

Krayer, A., Ingledew, D., \& Iphofen, R. (2008). Social comparison and body image in adolescence: A grounded theory approach. Health Education Research, 23(5), 892-903. doi: 10.1093/her/cym076

Leahey, T., Crowther, J., \& Mickelson, K. (2007). The frequency, nature, and effects of naturally occurring appearance-focused social comparisons. Behavior Therapy, 38 (2), 132-143. doi:10.1016/j.beth.2006.06.004

Leary, M., Tambor, E., Terdal, S., \& Downs, D. (1995). Self-esteem as an interpersonal monitor: The sociometer hypothesis. Journal of Personality Psychology, 68, 518-530. doi: 10.1037/00223514.68.3.518

Lev-Ari, L., Baumgarten-Katz, I., \& Zohar, A. H. (2014). Show me your friends, and I shall show you who you are: the way attachment and social comparisons influence body dissatisfaction. European Eating Disorders Review, 22(6), 463-469.

Lins, S., \& Poeschl, G. (2015). Gastar dinheiro em roupas no shopping: Os significados de "comprar" para adolescentes Brasileiros e Portugueses. Trends in Psychology, 23 (2), 355-369. doi: 10.9788/tp2015.2-06

Lorenzi-Cioldi, F. (1988). Individus dominants et groupes dominés. Images masculines et féminines. Grenoble: Presses Universitaires.

Marôco, J. (2014). Análise de equações estruturais, fundamentos teóricos, software \& aplicações (2 ed.). Pêro Pinheiro: Report Number.

Marsh, H., \& Parker, J. (1984). Determinants of student self-concept: Is it better to be a relatively large fish in a small pond even if you don't learn to swim as well? Journal of Personality \& Social Psychology, 47, 213-231. doi: 10.1037/0022-3514.47.1.213

Morse, S., \& Gergen, K. J. (1970). Social comparison, self-consistency, and the concept of self. Journal of Personality and Social Psychology, 16, 148-156. doi: 10.1037/h0029862 
Mueller, A., Pearson, J., Muller, C., Frank, K., \& Turner, A. (2010). Sizing up peers: adolescent girls' weight control and social comparison in the school context. Journal of Health and Social Behavior, 51(1) 64-78. doi: 10.1177/0022146509361191

Myers, T. A., \& Crowther, J. H. (2009). Social comparison as a predictor of body dissatisfaction: A metaanalytic review. Journal of Abnormal Psychology, 118(4), 683-698. doi:10.1037/a0016763

Newman, B., Lohman, B., \& Newman, P. (2007). Peer group membership and a sense of belonging: Their relationship to adolescent behaviour problems. Adolescence, 42, 241-263.

Norvilitis, J., \& Mendes-Da-Silva, W. (2013). Attitudes toward credit and finances among college students in Brazil and the United States. Journal of Business Theory and Practice, 1(1), 132-151.

Norvilitis, J., \& Yingmei, M. (2013). Attitudes towards credit and finances among college students in China and the United States. International Journal of Psychology, 48 (3), 389-398. doi: 10.1080/00207594.2011.645486

Nunnally, J. C. (1978). Psychometric theory (2nd ed.). New York: McGraw Hill.

Palmonari, A., Pombeni, M., \& Kirchler, E. (1992). Evolution of the self-concept in adolescence and social categorization processes. European Review of Social Psychology, 3, 285-308. doi: 10.1080/14792779243000096

Pasquali, L. (2005). Análise fatorial para pesquisadores. Brasília: LabPAM.

Paxton, S., Schutz, H., Wertheim, E., \& Muir, S. (1999). Friendship clique and peer influences on body image concerns, dietary restraint, extreme weight-loss behaviors, and binge eating in adolescent girls. Journal of Abnormal Psychology, 108, 255-266. doi: 10.1037/0021-843X.108.2.255

Petersen, S., Taube, K., Lehmann, K., Bergh ${ }^{1}$ and, O., \& Leupoldt, A. (2012). Social comparison and anxious mood in pulmonary rehabilitation: The role of cognitive focus. British Journal of Health Psychology, 17(3) 463-476. doi: 10.1111/j.2044-8287.2011.02048.x

Piko, B., \& Gibbons, F. (2008). Behavioral and psychosocial influences of risk perception among Hungarian adolescents. International Journal of Public Health, 53, 131-138. doi: 10.1007/s000-007054-9

Ponchio, M., Aranha, F., \& Todd, S. (2007). Estudo exploratório do construto de materialismo no contexto de consumidores de baixa renda do município de São Paulo. Revistas Gerenciais, 6(1), 13-21.

Richins, M. (2004). The material values scale: measurement properties and development of a short form. Journal of Consumer Research, 31(1), 209-219. doi: 10.1086/383436

Ruiter, M., \& Sheri, J. (2015). Mania risk and creativity: A multi-method study of the role of motivation. Journal of Affective Disorders, 170 (1), 52-58. doi: 10.1016/j.jad.2014.08.049

Santos, A. (2010). Inflexibilidade psicológica: validação do questionário de evitamento experiencial e fusão cognitiva para adolescentes $A F Q-Y$. (Dissertação de Mestrado em Psicologia Clínica), Instituto Miguel Torga, Coimbra.

Santos, C., \& Fernandes, D. (2006). Avaliação da escala Consumer Style Inventory (CSI) no contexto brasileiro. RAM - Revista de Administração Mackenzie, 7(2), 11-38.

Schumacker, R. E., \& Lomax, R. G. (2004). A beginner's guide to structural equation modelling. Mahwah, New Jersey: Lawrence Erlbaum Associates.

Segabinazi, J., Giacomoni, C., Dias, A., Teixeira, M., \& Moraes, D. (2010). Desenvolvimento e validação preliminar de uma escala multidimensional de satisfação de vida para adolescentes. Psicologia: Teoria e Pesquisa, 26(4), 653-659. doi: 10.1590/S0102-37722010000400009

Sproles, G., \& Kendall, E. (1986). A methodology for profiling consumers' decision-making styles. Journal of Consumer Affairs, 20(2), 267-279. doi: 10.1111/j.1745-6606.1986.tb00382.x

Strahan, E., Wilson, A., Cressman., K., \& Buote, V. (2006). Comparing to perfection: How cultural norms for appearance affect social comparisons and self-image. Body image 3, 211-227. doi: 10.1016/j.bodyim.2006.07.004

Suls, J., \& Miller, R. (1977). Social comparison processes: Theoretical and empirical perspectives. Washington: Hemisphere.

Taylor, S., \& Lobel, M. (1989). Social comparison activity under threat: downward evaluation and upward contacts. Psychological Review, 96(4), 569-575. doi: 10.1037/0033-295x.96.4.569

Twenge, J., \& Kasser, T. (2013). Generational changes in materialism and work centrality, 1976-2007: Associations with temporal changes in societal insecurity and materialistic role-modeling. Personality and Social Psychology Bulletin, 39, 883-897. doi: 10.1177/0146167213484586

Van der Zee, K., Oldersma, F., Buunk, B., \& Bos, D. (1998). Social comparison preferences among cancer patients as related to neuroticism and social comparison orientation. Journal of Personality and Social Psychology, 75, 801-810. doi: 10.1037/0022-3514.75.3.801

Wheeler, L., \& Miyake, K. (1992). Social comparison in everyday life. Journal of Personality and Social Psychology, 62, 760-773. doi: 10.1037/0022-3514.62.5.760 
White, J.B., Langer, E. J., Yariv, L., \& Welch IV, J.C. (2006). Frequent social comparisons and destructive emotions and behaviors: The dark side of social comparisons. Journal of Adult Development, 13(1), 36-44. doi: 10.1007/s10804-006-9005-0

Wood, J. (1989). Theory and research concerning social comparisons of personal attributes. Psychological Bulletin, 106, 231-248. doi: 10.1037/0033-2909.106.2.231

Wood, J., Taylor, S., \& Lichtman, R. (1985). Social comparison in adjustment to breast cancer. Journal of Personality \& Social Psychology, 49, 1169-1183. doi: 10.1037/0022-3514.49.5.1169

Xavier, A., Cunha, M., Gouveia, J., \& Medeiros (2014). Psychometric study of the adolescent social comparison scale-revised. Revista de Saúde Pública, 48 (n.esp), 190-281.

\section{ANEXO \\ Escala de Orientação para a Comparação Social (INCOM) para o contexto de adolescentes portugueses}

A maioria das pessoas compara-se, de vez em quando, com as outras. Elas podem comparar os seus sentimentos, opiniões, capacidades e/ou a sua situação com as das outras. Não existe particularmente nada de bom ou de mau neste tipo de comparações e algumas pessoas fazem-no com mais frequência do que outras.

As perguntas a seguir tentam determinar com que frequência te comparas com os outros e como te sentes ao realizar essas comparações. Para isso é necessário, por favor, que marques com um 'x' a opção da resposta escolhida em cada item.

\begin{tabular}{|c|c|c|c|c|c|c|}
\hline $\mathbf{1}$ & $\mathbf{2}$ & $\mathbf{3}$ & $\mathbf{4}$ & $\mathbf{5}$ & $\mathbf{6}$ \\
$\begin{array}{c}\text { Discordo } \\
\text { Totalmente }\end{array}$ & $\begin{array}{c}\text { Discordo } \\
\text { Muito }\end{array}$ & $\begin{array}{c}\text { Discordo } \\
\text { Pouco }\end{array}$ & $\begin{array}{c}\mathbf{7} \\
\text { Indiferente }\end{array}$ & $\begin{array}{c}\text { Concordo } \\
\text { Pouco }\end{array}$ & $\begin{array}{c}\text { Concordo } \\
\text { Muito }\end{array}$ \\
Totalmente \\
\hline
\end{tabular}

\begin{tabular}{|c|c|c|c|c|c|c|c|}
\hline $\begin{array}{l}\text { Comparo como as pessoas mais próximas a mim, (Ex. Família, amigos, namorado(a) agem com as } \\
\text { outras. }\end{array}$ & 1 & 2 & 3 & 4 & 5 & 6 & 7 \\
\hline $\begin{array}{l}\text { Presto sempre muita atenção ao modo como faço as coisas, comparando-as com o modo como os } \\
\text { outros as fazem. }\end{array}$ & 1 & 2 & 3 & 4 & 5 & 6 & 7 \\
\hline Se quero saber se o que estou a fazer está bem, comparo o que a faço com o que os outros fazem. & 1 & 2 & 3 & 4 & 5 & 6 & 7 \\
\hline $\begin{array}{l}\text { Comparo o meu desenvolvimento social (por exemplo: habilidades sociais, popularidade) em relação } \\
\text { às outras pessoas. }\end{array}$ & 1 & 2 & 3 & 4 & 5 & 6 & 7 \\
\hline Não sou uma pessoa que se compara com as outras. & 1 & 2 & 3 & 4 & 5 & 6 & 7 \\
\hline Comparo-me com os outros em relação ao que tenho conquistado na vida. & 1 & 2 & 3 & 4 & 5 & 6 & 7 \\
\hline Gosto de conversar com os outros sobre as opiniões e experiências em comum. & 1 & 2 & 3 & 4 & 5 & 6 & 7 \\
\hline Tento saber o que os outros pensam quando têm problemas semelhantes aos meus. & 1 & 2 & 3 & 4 & 5 & 6 & 7 \\
\hline Gosto sempre de saber o que os outros fariam no meu lugar. & 1 & 2 & 3 & 4 & 5 & 6 & 7 \\
\hline Se quero saber mais sobre algo, tento saber o que os outros pensam sobre isso. & 1 & 2 & 3 & 4 & 5 & 6 & 7 \\
\hline Nunca comparo a minha condição de vida com a das outras pessoas. & 1 & 2 & 3 & 4 & 5 & 6 & 7 \\
\hline
\end{tabular}

Historial do artigo

Recebido 21/07/2015

Aceite $\quad 09 / 05 / 2016$

Publicado $\quad 06 / 2016$ 
INCOM para o contexto de adolescentes portugueses 